\title{
Long-periodic precession parametrization
}

\author{
Jan Vondrák \\ Astronomical Institute, Academy of Sciences of the Czech Republic, \\ Boční II, 14131 Prague 4, Czech Republic \\ email: vondrak@ig.cas.cz
}

Abstract. We discuss aspects of long-periodic precession parametrization.

Keywords. methods: analytical, astrometry, reference systems, celestial mechanics, ephemerides

Both old (IAU1976) and newly adopted (IAU2006) parametrization of precession is based on polynomial developments of the associated angles. Such developments are designed to achieve very high precision for several centuries around the central epoch, J2000. However, when extrapolated to more distant epochs, they start to rapidly diverge from a more realistic long-term predictions coming from a numerical integration of the motion of the rotating Earth and the bodies of the solar system.

The aim of this presentation is to propose another parametrization of precession, based on long-periodic functions of time rather than polynomials, which would yield results with precision approaching that of IAU2006 for epochs close to J2000 (up to a few thousand years), and a good fit to numerical integration for much longer time intervals (up to several hundred centuries).

It is demonstrated that such solution is possible with relatively low number of periodic terms, both for the precession of the ecliptic (six periodic terms with periods ranging from 500 to 2300 centuries) and the general precession / obliquity (ten periodic terms with periods ranging from 200 to 4000 centuries).

This study is a preparatory phase for the prepared work by N. Capitaine, P.T. Wallace and J. Vondrák that should lead to a next generation of precession model. 